\title{
SOME PROPERTIES OF QUASIUNIFORM MULTIFUNCTION SPACES
}

\author{
JILING CAO, IVAN L. REILLY and SALVADOR ROMAGUERA
}

(Received 22 November 1996; revised 11 July 1997)

Communicated by J. A. Hillman

\begin{abstract}
The aim of this paper is to explore some properties of quasiuniform multifunction spaces. Various kinds of completeness of the quasiuniform multifunction space $\left(Y^{m X}, \mathscr{U}_{m X}\right)$ are characterized in terms of suitable properties of the range space $(Y, \mathscr{U})$. We also discuss the local compactness of quasiuniform multifunction spaces. By using the notion of small-set symmetry, the classic result of Hunsaker and Naimpally is extended to the quasiuniform setting.
\end{abstract}

1991 Mathematics subject classification (Amer. Math. Soc.): primary 54C35; secondary 54C60, $54 \mathrm{E} 15$. Keywords and phrases: Quasiuniform, multifunction, complete, stable filter, Vietoris topology..

\section{Introduction}

Spaces of functions have been used since the late 19th century to study convergence of sequences of functions. Nowadays, function spaces play an important role in both topology and analysis. Around the 1950s, a group of famous mathematicians including Arens, Fox, Myers and Kelley, established the theory of uniform function spaces. A good survey on this topic is [11]. Since then, several attempts to extend this theory have been made. First of all, Smithson $[23,24]$ successfully generalized some results of [11] to the case of multifunctions. Naimpally [18] and Seyedin [22] considered the quasiuniform case. But they seem to have been unsuccessful: in fact, the main results of [18] and [22] are incorrect as shown in [2]. With the development of the theory of quasiuniform spaces, this topic has been re-considered in the last years by several authors (see $[2,3,12,14,15,19]$, for example).

Recently Künzi and Ryser [16] investigated the Bourbaki quasiuniformity. Since

The work of the first two authors has been supported by a grant from the University of Auckland Research Committee, and the third author acknowledges the support of the DGES, grant PB 95-0737.

(C) 1998 Australian Mathematical Society $0263-6115 / 98 \$ \mathrm{~A} 2.00+0.00$ 
hyperspaces, multifunctions and function spaces interact with each other, the main purpose of this paper is to study some properties of quasiuniform multifunction spaces by using both the hyperspace approach and the function space approach. Thus, by means of some results in $[14,15,16]$, we provide characterizations of various kinds of completeness of the quasiuniform function space $\left(Y^{m X}, \mathscr{U}_{m X}\right)$ in Section 3. The local compactness of families of multifunctions in quasiuniform function spaces is discussed in Section 4. By using the notion of small-set symmetry introduced by Fletcher and Hunsaker [8] recently, the classic result of Hunsaker and Naimpally [10] is extended to the quasiuniform setting.

\section{Preliminaries}

Our basic reference for quasiuniform and quasi-pseudometric spaces is [9]. Let $(Y, \mathscr{U})$ be a quasiuniform space. The conjugate quasiuniformity of $\mathscr{U}$ is denoted by $\mathscr{U}^{-1}$. The coarsest uniformity containing $\mathscr{U}$ is denoted by $\mathscr{U}^{s}\left(=\mathscr{U} \vee \mathscr{U}^{-1}\right)$. Moreover, $\mathscr{T}(\mathscr{U})$ and $\mathscr{T}\left(\mathscr{U}^{-1}\right)$ will denote the topologies on $Y$ induced by $\mathscr{U}$ and $\mathscr{U}^{-1}$, respectively. Let $\mathscr{P}_{0}(Y)$ be the collection of all nonempty subsets of $Y$. Then $\left(\mathscr{P}_{0}(Y), \mathscr{U}_{*}\right)$ stands for the hyperspace of $\mathscr{P}_{0}(Y)$ with $\mathscr{U}_{*}$ the Bourbaki quasiuniformity induced by $\mathscr{U}[1,16]$. A quasiuniform space $(Y, \mathscr{U})$ is called bicomplete [9] if the uniformity $\mathscr{U}^{s}$ is complete. Furthermore, $(Y, \mathscr{U})$ is called half-complete [6] if each $\mathscr{U}^{s}$-Cauchy filter is $\mathscr{T}(\mathscr{U})$-convergent. A filter on $(Y, \mathscr{U})$ is called right $K$-Cauchy if for each $U \in \mathscr{U}$ there is $F \in \mathscr{F}$ such that $U^{-1}(x) \in \mathscr{F}$ for all $x \in F$, and $(Y, \mathscr{U})$ is called right $K$-complete if each right $K$-Cauchy filter on it $\mathscr{T}(\mathscr{U})$-converges [21]. In order to simplify the proofs of some results we shall use certain convergent nets instead of filters. To this end, we recall that a net $\left\{x_{\alpha}, \alpha \in \Lambda\right\}$ in $(Y, \mathscr{U})$ is right $K$-Cauchy [14] if for each $U \in \mathscr{U}$ there is a $\gamma \in \Lambda$ such that $\left(x_{\alpha}, x_{\beta}\right) \in U$ whenever $\gamma \leq \beta \leq \alpha$. It is shown in [14] that a quasiuniform space $(Y, \mathscr{U})$ is right $K$-complete if and only if each right $K$-Cauchy net in $(Y, \mathscr{U})$ is $\mathscr{T}(\mathscr{U})$-convergent.

Let $X$ and $Y$ be two topological spaces. We recall three topologies defined on $\mathscr{P}_{0}(Y)$ (see Michael [17]). The collection of all sets of the form $M^{+}=\left\{B \in \mathscr{P}_{0}(Y): B \subseteq M\right\}$ where $M$ is open in $Y$, is a base for the upper Vietoris topology. The collection of all sets of the form $M^{-}=\left\{B \in \mathscr{P}_{0}(Y): B \cap M \neq \emptyset\right\}$ where $M$ is open in $Y$, is a base for the lower Vietoris topology. The Vietoris topology is the supremum of the upper Vietoris topology and the lower Vietoris topology. A multifunction (or multi-valued function, set-valued function, relation) $F: X \rightarrow Y$ is a point-to-set correspondence from $X$ to $Y$ such that $F(x) \neq \emptyset$ for each $x \in X$. If $F(x)$ is a compact subset of $Y$ for each $x \in X$, then $F$ is called compact-valued. Moreover, $F$ is continuous if and only if $F: X \rightarrow \mathscr{P}_{0}(Y)$ is continuous relative to the Vietoris topology on $\mathscr{P}_{0}(Y)$. 
Let $\mathscr{F}$ be a family of multifunctions from $X$ to $Y$. The pointwise topology $\mathscr{T}_{\mathscr{P}}$ [23] on $\mathscr{F}$ has as a subbase the sets of the form $\{F \in \mathscr{F}: F(x) \subseteq M\}$ and $\{F \in \mathscr{F}: F(x) \cap N \neq \emptyset\}$ where $x \in X$ and $M, N$ are open in $Y$. If each $F \in \mathscr{F}$ is identified with its graph $\{(x, y) \in X \times Y: x \in X, y \in F(x)\}$ in the product space $X \times Y$, then the topology on $\mathscr{F}$ induced by the Vietoris topology (respectively the upper Vietoris topology, the lower Vietoris topology) on $\mathscr{P}_{0}(X \times Y)$ is denoted by $\mathscr{T}_{\mathcal{V}}$ (respectively $\mathscr{T}_{\mathcal{Y}}^{+}, \mathscr{T}_{\mathcal{Y}}^{-}$).

Let $Y^{m X}$ (respectively $Y^{X}$ ) be the set of all multifunctions (respectively functions) from a nonempty set $X$ to a quasiuniform space $(Y, \mathscr{U})$. For each $U \in \mathscr{U}$, define

$$
W(U)=\left\{(F, H): H(x) \subseteq U(F(x)) \text { and } F(x) \subseteq U^{-1}(H(x)) \text { for all } x \in X\right\} .
$$

Then $\{W(U): U \in \mathscr{U}\}$ is a base for a quasiuniformity $\mathscr{U}_{m X}$ on $Y^{m X}$ called the quasiuniformity of quasiuniform convergence (on $Y^{m X}$ ). The topology induced by $\mathscr{U}_{m X}$ on $Y^{m X}$, denoted by $\mathscr{T}_{\mathscr{Q}}$, is called the topology of quasiuniform convergence (see [2]). The restriction of $\mathscr{U}_{m X}$ on $Y^{X}$, denoted by $\mathscr{U}_{X}$, is the quasiuniformity of quasiuniform convergence (on $Y^{X}$ ) defined in [18]. A simple observation is that $Y^{m X}=\left(\mathscr{P}_{0}(Y)\right)^{X}$ and $\mathscr{U}_{m X}=\left(\mathscr{U}_{*}\right)_{X}$. Moreover, if $(Y, \mathscr{U})$ is a uniform space, then $\mathscr{U}_{m X}$ is exactly the uniformity of uniform convergence on $Y^{m X}$ defined in [24]. Let $(Y, d)$ be a bounded quasi-pseudometric space. Then the quasi-pseudometric $D$ defined on $Y^{X}$ by $D(f, g)=\sup \{d(f(x), g(x)): x \in X\}$ induces the quasiuniformity of quasiuniform convergence of $\mathscr{U}_{d}$. Let $d_{*}$ be the Hausdorff quasi-pseudometric on $\mathscr{P}_{0}(Y)$ defined by $d_{*}(A, B)=\max \left\{\sup _{b \in B} d(A, b), \sup _{a \in A} d(a, B)\right\}$ for all $A, B \in \mathscr{P}_{0}(Y)$. Note that $d_{*}$ induces on $\mathscr{P}_{0}(Y)$ the Bourbaki quasiuniformity of the quasi-uniform space $\left(Y, \mathscr{U}_{d}\right)$ (see [1]). Since $Y^{m X}=\left(\mathscr{P}_{0}(Y)\right)^{X}$, the quasi-pseudometric $D^{m}$ on $Y^{m X}$ defined by $D^{m}(F, G)=\sup \left\{d_{*}(F(x), G(x)): x \in X\right\}$ induces on $Y^{m X}$ the quasiuniformity of quasiuniform convergence of $\mathscr{U}_{d_{*}}$, that is, $\left(\mathscr{U}_{d}\right)_{m X}$.

\section{Completeness of quasiuniform multifunction spaces}

The proofs of the following two lemmas are straightforward, so we omit them.

LEMMA 3.1. Let $X$ be a non-empty set and $(Y, \mathscr{U})$ be a quasiuniform space. Then the function $\Phi:\left(Y^{m X}, \mathscr{U}_{m X}\right) \rightarrow\left(\mathscr{P}_{0}(Y), \mathscr{U}_{*}\right)$ defined by $\Phi(F)=\cup_{x \in X} F(x)$ is quasiuniformly continuous.

LEMMA 3.2. Let $X$ be a non-empty set and $(Y, \mathscr{U})$ be a quasiuniform space. For each $A \in \mathscr{P}_{0}(Y)$, define $F_{A} \in Y^{m X}$ by $F_{A}(x)=A$ for all $x \in X$. Then $\varphi:\left(\mathscr{P}_{0}(Y), \mathscr{U}_{*}\right) \rightarrow\left(Y^{m X}, \mathscr{U}_{m X}\right)$ defined by $\varphi(A)=F_{A}$ is quasiuniformly continuous.

It is quite obvious that $\Phi(\varphi(A))=A$ for each $A \in \mathscr{P}_{0}(Y)$. 
LEMMA 3.3 ([14]). Let $X$ be a non-empty set and $(Y, \mathscr{U})$ be a quasiuniform space. Then

(1) $\left(Y^{X}, \mathscr{U}_{X}\right)$ is right $K$-complete if and only if $(Y, \mathscr{U})$ is right $K$-complete.

(2) $\left(Y^{X}, \mathscr{U}_{X}\right)$ is bicomplete if and only if $(Y, \mathscr{U})$ is bicomplete.

Let $(Y, \mathscr{U})$ be a quasiuniform space. Recall that a filter on $(Y, \mathscr{U})$ is stable (respectively doubly stable) provided that for any $U \in \mathscr{U}, \cap_{F \in \mathscr{F}} U(F)$ (respectively $\left.\cap_{F \in \mathscr{F}}\left(U(F) \cap U^{-1}(F)\right)\right)$ belongs to $\mathscr{F}$ [5]. Moreover, a point $y \in Y$ is called a double cluster point of a filter $\mathscr{F}$ on $(Y, \mathscr{U})$ if $y \in \cap_{F \in \mathscr{F}}(\mathscr{T}(\mathscr{U}) \mathrm{cl} F) \cap\left(\mathscr{T}\left(\mathscr{U}^{-1}\right) \mathrm{cl} F\right)$ [16].

LEMMA 3.4 ([16]). Let $(Y, \mathscr{U})$ be a quasiuniform space. Then

(1) $\left(\mathscr{P}_{0}(Y), \mathscr{U}_{*}\right)$ is right $K$-complete if and only if each stable filter on $(Y, \mathscr{U})$ has a cluster point.

(2) $\left(\mathscr{P}_{0}(Y), \mathscr{U}_{*}\right)$ is bicomplete if and only if for each doubly stable filter $\mathscr{F}$ on $(Y, \mathscr{U})$ and each $U \in \mathscr{U}$ there is an $F \in \mathscr{F}$ such that $F \subseteq U(C) \cap U^{-1}(C)$, where $C$ denotes the set of all double cluster points of $\mathscr{F}$.

As an immediate consequence of lemmas described above, we have the following

Proposition 3.5. Let $X$ be a non-empty set and $(Y, \mathscr{U})$ be a quasiuniform space. Then

(1) $\left(Y^{m X}, \mathscr{U}_{m X}\right)$ is right $K$-complete if and only if every stable filter on $(Y, \mathscr{U})$ has a cluster point.

(2) $\left(Y^{m X}, \mathscr{U}_{m X}\right)$ is bicomplete if and only iffor each doubly stable filter $\mathscr{F}$ on $(Y, \mathscr{U})$ and each $U \in \mathscr{U}$ there is an $F \in \mathscr{F}$ such that $F \subseteq U(C) \cap U^{-1}(C)$, where $C$ denotes the set of all double cluster points of $\mathscr{F}$.

PROPOSITION 3.6. Let $X$ be a non-empty set and $(Y, \mathscr{U})$ be a quasiuniform space. Then $\left(Y^{m X}, \mathscr{U}_{m X}\right)$ is half-complete if and only if for any doubly stable filter $\mathscr{F}$ on $(Y, \mathscr{U})$ and each $U \in \mathscr{U}$ there is an $F \in \mathscr{F}$ such that $F \subseteq U(C)$, where $C$ denotes the set of all cluster points of $\mathscr{F}$.

PROOF. Suppose that $\left(Y^{m X}, \mathscr{U}_{m X}\right)$ is half-complete. Let $\left\{A_{\alpha}: \alpha \in \Lambda\right\}$ be a $\left(\mathscr{U}_{*}\right)^{s}$ Cauchy net in $\left(\mathscr{P}_{0}(Y), \mathscr{U}_{*}\right)$. By Lemma $3.2,\left\{\varphi\left(A_{\alpha}\right): \alpha \in \Lambda\right\}$ is a $\left(\mathscr{U}_{m X}\right)^{s}$-Cauchy net in $\left(Y^{m X}, \mathscr{U}_{m X}\right)$, so it is convergent to some $F \in Y^{m X}$. By Lemma 3.1, $\left\{A_{\alpha}: \alpha \in \Lambda\right\}$ is convergent in $\left(\mathscr{P}_{0}(Y), \mathscr{U}_{*}\right)$. Therefore $\left(\mathscr{P}_{0}(Y), \mathscr{U}_{*}\right)$ is half-complete. Let $\mathscr{F}$ be a doubly stable filter on $(Y, \mathscr{U})$ and $U \in \mathscr{U}$, and let $C$ denote the set of all cluster points of $\mathscr{F}$. Then the net $(F)_{F \in(\mathscr{F} . \supseteq)}$ is $\left(\mathscr{U}_{*}\right)^{s}$-Cauchy, so it converges to some $B \in \mathscr{P}_{0}(Y)$. It is easily seen that $B \subseteq C$. Since $(F)_{F \in(\mathscr{F} . \supseteq)}$ converges to $B$, then for each $U \in \mathscr{U}$ there exists an $F \in \mathscr{F}$ such that $F \subseteq U(B)$. Hence $F \subseteq U(C)$. 
Conversely, suppose that each doubly stable filter on $(Y, \mathscr{U})$ satisfies the given condition. We shall show that $\left(\mathscr{P}_{0}(Y), \mathscr{U}_{*}\right)$ is half-complete. To this end, let $\left\{A_{\alpha}: \alpha \in\right.$ $\Lambda\}$ be a $\left(\mathscr{U}_{*}\right)^{s}$-Cauchy net in $\mathscr{P}_{0}(Y)$. Consider the filter $\mathscr{F}=$ fil $\left\{F_{\alpha}: \alpha \in \Lambda\right\}$ on $Y$ where $F_{\alpha}=\cup_{\beta \geq \alpha} A_{\beta}$ for all $\alpha \in \Lambda$. Then $\mathscr{F}$ is a doubly stable filter on $(Y, \mathscr{U})$. Let $C$ be the set of all cluster points of $\mathscr{F}$, and let $U \in \mathscr{U}$. Choose $V \in \mathscr{U}$ with $V^{2} \subseteq U$. By hypothesis there is an $\alpha_{V} \in \Lambda$ such that $F_{\alpha_{1}} \subseteq V(C)$. Without loss of generality, we can suppose that $A_{\alpha} \subseteq V\left(A_{\beta}\right)$ and $A_{\beta} \subseteq V^{-1}\left(A_{\alpha}\right)$ for all $\alpha, \beta \geq \alpha_{V}$. Then for all $\alpha \geq \alpha_{V}, A_{\alpha} \subseteq F_{\alpha_{1}} \subseteq V(C)$. Moreover, $C \subseteq U^{-1}\left(A_{\alpha}\right)$ for all $\alpha \geq \alpha_{V}$. In fact, if $x \in C$, then $V(x) \cap F_{\alpha} \neq \emptyset$. It follows that $V(x) \cap A_{\beta} \neq \emptyset$ for some $\beta \geq \alpha$ and thus $x \in V^{-1}\left(A_{\beta}\right) \subseteq V^{-1}\left(V^{-1}\left(A_{\alpha}\right)\right) \subseteq U^{-1}\left(A_{\alpha}\right)$. This proves that $\left(\mathscr{P}_{0}(Y), \mathscr{U}_{*}\right)$ is half-complete. Since $Y^{m X}=\left(\mathscr{P}_{0}(Y)\right)^{X}$ and $\mathscr{U}_{m X}=\left(\mathscr{U}_{*}\right)_{X}$, we deduce from [15, Theorem $3.1(1)]$ that $\left(Y^{m X}, \mathscr{U}_{n^{\prime}}\right)$ is half-complete.

A sequence $\left\{y_{k}: k \in \omega\right\}$ in a quasi-pseudometric space $(Y, d)$ is called right $K$ Cauchy if for each $\varepsilon>0$ there is an integer $k \in \omega$ such that $d\left(y_{n}, y_{m}\right)<\varepsilon$ whenever $n \geq m \geq k$. A quasi-pseudometric space $(Y, d)$ is called right $K$-sequentially complete if each right $K$-Cauchy sequence converges [20]. The following result, whose proof is based on the classical argument, is a quasi-metric analogue to Lemma 3.3 (1).

PROPOSITION 3.7. Let $X$ be a non-empty set and $(Y, d)$ be a bounded quasipseudometric space. Then $\left(Y^{X}, D\right)$ is right $K$-sequentially complete if and only if $(Y, d)$ is right $K$-sequentially complete.

PROOF. Suppose that $\left(Y^{X}, D\right)$ is right $K$-sequentially complete. Let $\left\{y_{k}: k \in \omega\right\}$ be a right $K$-Cauchy sequence in $(Y, d)$. For each $k \in \omega$, define $f_{k}(x)=y_{k}$ for all $x \in X$. Obviously, $\left\{f_{k}: k \in \omega\right\}$ is a right $K$-Cauchy sequence in $\left(Y^{X}, D\right)$, so it converges to some $f \in Y^{X}$. It follows that $\left\{y_{k}: k \in \omega\right\}$ converges to $f(x)$ for any fixed $x \in X$. Therefore $(Y, d)$ is right $K$-sequentially complete.

Conversely, suppose that $(Y, d)$ is right $K$-sequentially complete. To show that $\left(Y^{X}, D\right)$ is right $K$-sequentially complete, let $\left\{f_{k}: k \in \omega\right\}$ be a right $K$-Cauchy sequence in $\left(Y^{X}, D\right)$. For each $x \in X,\left\{f_{k}(x): k \in \omega\right\}$ is a right $K$-Cauchy sequence in $(Y, d)$, so it converges to some point $y_{x}$. Then $f(x)=y_{x}$ defines a function $f \in Y^{X}$. Since $\left\{f_{k}: k \in \omega\right\}$ is right $K$-Cauchy in $\left(Y^{X}, D\right)$, for each $\varepsilon>0$ there is an integer $k \in \omega$ such that $D\left(f_{n}, f_{m}\right)<\varepsilon$ whenever $n \geq m \geq k$. For any $x \in X$, we have $d\left(f_{n}(x), f_{m}(x)\right)<\varepsilon$ whenever $n \geq m \geq k$, so $d\left(f(x), f_{n}(x)\right)<2 \varepsilon$ for all $m \geq k$. This implies that $D\left(f, f_{m}\right) \leq 2 \varepsilon$ for all $m \geq k$. Therefore $\left\{f_{k}: k \in \omega\right\}$ converges to $f$ in $\left(Y^{X}, D\right)$ which implies that $\left(Y^{X}, D\right)$ is right $K$-sequentially complete.

LEMMA 3.8 ([16]). Let $(Y, d)$ be a bounded quasi-pseudometric space. Then $(Y, d)$ is right $K$-sequentially complete if and only if $\left(\mathscr{P}_{0}(Y), d_{*}\right)$ is right $K$-sequentially complete. 
COROLLARY 3.9. Let $X$ be a non-empty set and $(Y, d)$ be a bounded quasi-pseudometric space. Then $\left(Y^{m X}, D^{m}\right)$ is right $K$-sequentially complete if and only if $(Y, d)$ is right $K$-sequentially complete.

REMARK. Note that [16, Example 3 and Proposition 6] show that Corollary 3.9 cannot be extended to the case that $(Y, d)$ is right $K$-complete.

\section{Local compactness of families of multifunctions}

LEMMA 4.1. Let $X$ be a compact space and $(Y, \mathscr{U})$ be a quasiuniform space. If $\mathscr{F}$ is a family of continuous and compact-valued multifunctions from $X$ to $(Y, \mathscr{U})$, then $\mathscr{T}_{\mathscr{Y}} \leq \mathscr{T}_{\mathscr{Q}}$ on $\mathscr{F}$.

PRoOF. We first show $\mathscr{T}_{\bar{y}}^{-} \leq \mathscr{T}_{\mathscr{Q}}$. Let $F \in M^{-}$where $M$ is open in $X \times Y$. Then there exist $x \in X$ and $y \in F(x)$ with $(x, y) \in M$. Choose a $V \in \mathscr{U}$ such that $\{x\} \times V(y) \subseteq M$. For any $H \in W(V)(F)$, there is a $y^{\prime} \in H(x)$ with $y^{\prime} \in V(y)$. Thus $H \in M^{-}$. It follows that $\mathscr{T}_{V}^{-} \leq \mathscr{T}_{\mathscr{Q}}$. Let $F \in M^{+}$where $M$ is open in $X \times Y$. By Wallace's theorem [7, p. 186], for each $x \in X$ there exist an open neighbourhood $G_{x}$ of $x$ and a $V_{x} \in \mathscr{U}$ such that $G_{x} \times V_{x}^{2}(F(x)) \subseteq M$. Since $F$ is continuous, we can assume that $F\left(G_{x}\right) \subseteq V_{x}(F(x))$. Since $X$ is compact, there is a finite subset $A$ of $X$ such that $X=\cup_{x \in A} G_{x}$. Let $V=\cap_{x \in A} V_{x}$. We claim that $W(V)(F) \subseteq M^{+}$. In fact, for any $H \in W(V)(F)$ and any $x \in X$, choose $a \in A$ such that $x \in G_{a}$. Then $F(x) \subseteq V_{a}(F(a))$. Consequently, $H(x) \subseteq V(F(x)) \subseteq V_{a}^{2}(F(a))$. It follows that $H \in M^{+}$. Therefore $\mathscr{T}_{\mathscr{V}}^{+} \leq \mathscr{T}_{\mathscr{Q}}$.

DEFINITION 1 ([4]). Let $X$ be a space and $(Y, \mathscr{U})$ be a quasiuniform space. A subset $\mathscr{F} \in Y^{m X}$ is called quasi-equicontinuous if for each $x \in X$ and each $U \in \mathscr{U}$ there is a neighbourhood $G$ of $x$ such that for all $F \in \mathscr{F}$

(1) $F(G) \subseteq U(F(x))$, and

(2) $F(z) \cap U(y) \neq \emptyset$ for each $z \in G$ and each $y \in F(x)$.

A quasiuniform space $(Y, \mathscr{U})$ is called small-set symmetric [8] provided that for each $U \in \mathscr{U}$ and each open set $A \subseteq Y$ we have $\mathscr{T}(\mathscr{U}) \mathrm{cl} A \subseteq U(A)$. A well-known fact is that $(Y, \mathscr{U})$ is small-set symmetric if and only if $\mathscr{T}\left(\mathscr{U}^{-1}\right) \subseteq \mathscr{T}(\mathscr{U})$ [13].

PROPOSITION 4.2. Let $X$ be a $T_{1}$ compact space and $(Y, \mathscr{U})$ be a small-set symmetric quasiuniform space. If $\mathscr{F} \subseteq Y^{m X}$ is a family of quasi-equicontinuous and compact-valued multifunctions, then $\mathscr{T}_{\mathscr{P}}=\mathscr{T}_{\mathscr{Y}}=\mathscr{T}_{\mathscr{Q}}$ on $\mathscr{F}$. 
PROOF. Checking the proof of [10, Lemma 2.5], we see that no symmetry was used, so $\mathscr{T}_{\mathscr{P}} \leq \mathscr{T}_{\mathscr{Y}}$ on $\mathscr{F}$ follows. According to [4, Corollary 4.6] and the final remark in [4], $\mathscr{T}_{\mathscr{P}}=\mathscr{T}_{\mathscr{Q}}$. Following from Lemma $4.1, \mathscr{T}_{\mathscr{P}}=\mathscr{T}_{\mathscr{V}}=\mathscr{T}_{\mathscr{Q}}$ on $\mathscr{F}$.

To prove our main result in this section, we first extend both [12, Lemma 1] and $[24$, Lemma 6] as follows

PROPOSITION 4.3. Let $\mathscr{F}$ be a family of compact-valued multifunctions from a space $X$ to a small-set symmetric quasiuniform space $(Y, \mathscr{U})$. If $\mathscr{F}$ is quasi-equicontinuous, then $\mathscr{T}_{\mathscr{P}} \mathrm{cl} \mathscr{F}$, the $\mathscr{T}_{\mathscr{P}}$-closure of $\mathscr{F}$ in the space of all compact-valued multifunctions, is quasi-equicontinuous.

ProOF. For each $x \in X$ and each $U \in \mathscr{U}$, choose $V \in \mathscr{U}$ with $V^{4} \subseteq U$. Since $\mathscr{F}$ is quasi-equicontinuous, there exists a neighbourhood $G$ of $x$ such that for all $F \in \mathscr{F}$

(1) $F(G) \subseteq V(F(x))$,

(2) $F(z) \cap V(y) \neq \emptyset$, for any $y \in F(x)$ and $z \in G$.

Let $H \in \mathscr{T}_{\mathscr{P}} \mathrm{cl} \mathscr{F}$ and $z \in G$. Let $\left\{F_{\alpha}: \alpha \in \Lambda\right\} \subseteq \mathscr{F}$ be a net which $\mathscr{T}_{\mathscr{P}}$-converges to $H$. Since $H(x)$ is compact and $(Y, \mathscr{U})$ is small-set symmetric, there exists a finite subset $B$ of $H(x)$ such that $H(x) \subseteq \cup_{b \in B}\left(V(b) \cap V^{-1}(b)\right)$. Choose an $\alpha_{1} \in \Lambda$ such that $F_{\alpha}(x) \subseteq V(H(x))$ for all $\alpha \geq \alpha_{1}$ and $F_{\alpha}(x) \cap V(b) \neq \emptyset$ for all $\alpha \geq \alpha_{1}$ and $b \in B$. Hence $b \in V^{-1}\left(F_{\alpha}(x)\right)$ for all $b \in B$. So $H(x) \subseteq V^{-2}\left(F_{\alpha}(x)\right)$ for all $\alpha \geq \alpha_{1}$. On the other hand, by the compactness of $H(z)$, there exists a finite subset $A \subseteq H(z)$ such that $H(z) \subseteq \cup_{a \in A}\left[V(a) \cap V^{-1}(a)\right]$. Choose $\alpha_{2} \in D$ such that $F_{\alpha}(z) \subseteq \cup_{a \in A}\left[V(a) \cap V^{-1}(a)\right]$ and $F_{\alpha}(z) \cap\left[V(a) \cap V^{-1}(a)\right] \neq \emptyset$ for all $a \in A$ whenever $\alpha \geq \alpha_{2}$. Consequently, $H(z) \subseteq \cup_{a \in A} V(a) \subseteq V^{2}\left(F_{\alpha}(z)\right) \subseteq V^{4}(H(x)) \subseteq U(H(x))$ for any $\alpha \geq \max \left\{\alpha_{1}, \alpha_{2}\right\}$. Since $z$ is arbitrarily chosen, we have $H(G) \subseteq U(H(x))$. On the other hand, for any $y \in H(x)$, choose $r \in F_{\alpha}(x)$ with $r \in V^{2}(y)$ and $s \in V(r) \cap F_{\alpha}(z)$ for any $\alpha \geq \alpha_{2}$. Then $s \in V^{3}(y) \cap F_{\alpha}(z)$. Choose $a \in A$ such that $s \in V^{-1}(a)$. It follows that $a \in V^{4}(y) \cap H(z) \subseteq U(y) \cap H(z)$. Thus $H(z) \cap U(y) \neq \emptyset$. Since $H$ is arbitrarily chosen, $\mathscr{T}_{\mathscr{P}} \mathrm{cl} \mathscr{F}$ is quasi-equicontinuous.

COROLLARY 4.4 ([12]). Let $X$ be a space and let $(Y, \mathscr{U})$ be a small-set symmetric quasiuniform space. If $\mathscr{H}$ is a quasi-equicontinuous family of functions in $\mathscr{C}(X, Y)$ (the family of all continuous functions from $X$ to $Y$ ), then its $\mathscr{T}_{\mathscr{P}}$-closure $\mathscr{T}_{\mathscr{P}} \mathrm{cl} \mathscr{H}$ is also quasi-equicontinuous.

COROLLARY 4.5 ([24]). Let $\mathscr{F}$ be a family of compact-valued multifunctions from a space $X$ to a uniform space $(Y, \mathscr{U})$. If $\mathscr{F}$ is equicontinuous, then $\mathscr{T}_{\mathscr{P}} \mathrm{cl} \mathscr{F}$, the $\mathscr{T}_{\mathscr{P}}$-closure of $\mathscr{F}$ in the space of all compact-valued multifunctions, is equicontinuous. 
Proposition 4.6. Let $\mathscr{F}$ be a quasi-equicontinuous family of compact-valued multifunctions from a compact Hausdorff space $X$ into a small-set symmetric quasiuniform space $(Y, \mathscr{U})$. If $(Y, \mathscr{U})$ is locally compact, then $\mathscr{T}_{\mathscr{P}} \mathrm{cl} \mathscr{F}$, the $\mathscr{T}_{\mathscr{P}}$-closure of $\mathscr{F}$ in the space of all compact-valued multifunctions, is locally compact in $\left(Y^{m X}, \mathscr{U}_{m X}\right)$.

PROOF. Following Proposition 4.6, $\mathscr{T}_{\mathscr{P}} \mathrm{cl} \mathscr{F}$ is quasi-equicontinuous. By a proof similar to that of [10, Lemma 2.6], $\left(\mathscr{T}_{\mathscr{P}} \mathrm{cl} \mathscr{F}, \mathscr{T}_{Y}\right)$ is a closed subspace of $(\mathscr{K}(X \times$ $\left.Y), \mathscr{T}_{Y}\right)$, where $\mathscr{K}(X \times Y)$ is the family of all non-empty compact subsets of $X \times$ $Y$. Since $\left(\mathscr{K}(X \times Y), \mathscr{T}_{\mathscr{Y}}\right)$ is locally compact (see [17, Proposition 4.4.1]), so is $\left(\mathscr{T}_{\mathscr{P}} \mathrm{cl} \mathscr{F}, \mathscr{T}_{\mathscr{Y}}\right)$. By Proposition $4.3, \mathscr{T}_{\mathscr{P}}=\mathscr{T}_{\mathscr{Y}}=\mathscr{T}_{\mathscr{Q}}$ on $\mathscr{F}$. Therefore, $\mathscr{T}_{\mathscr{P}} \mathrm{cl} \mathscr{F}$ is locally compact in $\left(Y^{m X}, \mathscr{U}_{m X}\right)$.

COROLLARY 4.7 ([10]). Let $\mathscr{F}$ be an equicontinuous family of compact-valued multifunctions from a compact Hausdorff space $X$ to a locally compact Hausdorff uniform space $(Y, \mathscr{U})$. Let $\mathscr{T}_{\mathscr{P}} \mathrm{cl} \mathscr{F}$ be the $\mathscr{T}_{\mathscr{P}}$-closure of $\mathscr{F}$ in the space of all compact-valued multifunctions. Then $\mathscr{T}_{\mathscr{G}} \mathrm{cl} \mathscr{F}$ is locally compact in $\left(Y^{m X}, \mathscr{U}_{m X}\right)$.

\section{Acknowledgement}

The authors acknowledge the privilege of having seen [16] before its publication.

\section{References}

[1] G. Berthiaume, 'On quasiuniformities in hyperspaces', Proc. Amer: Math. Soc. 66 (1977), 335-343.

[2] J. Cao, 'Quasiuniform convergence for multifunctions', J. Math. Res. Exposition 14 (1994), 327331.

[3] —_, 'Answers to two questions of Papadopoulos', Questions Answers Gen. Topology 14 (1996), $111-116$.

[4] J. Cao and I. L. Reilly, 'Almost quasiuniform convergence for multifunctions', Ann. Soc. Math. Polon. Ser: I Comment. Math. Prace Mat. 36 (1996), 57-68.

[5] A. Császár, 'Extensions of quasi-uniformities', Acta Math. Hungar: 37 (1981), 121-145.

[6] J. Deák, 'On the coincidence of some notions of quasiuniform completeness defined by filter pairs', Studia. Sci. Math. Hungar: 26 (1991), 411-413.

[7] R. Engelking, General topology (Warszawa, 1977).

[8] P. Fletcher and W. N. Hunsaker, 'Symmetry conditions in terms of open sets', Topology Appl. 45 (1992), 39-47.

[9] P. Fletcher and W. Lindgren, Quasiuniform spaces, Lecture Notes in Pure and Appl. Math. 77. (Marcel Dekker, New York, 1982).

[10] W. N. Hunsaker and S. A. Naimpally, 'Local compactness of families of continuous point-compact relations', Pacific J. Math. 52 (1974), 101-105.

[11] J. L. Kelley, General topology (Van Nostrand, Princeton, 1955). 
[12] H. P. A. Künzi, 'On quasiuniform convergence and quiet spaces', Questions Answers Gen. Topology 12 (1994), 121-131.

[13] H. P. A. Künzi, M. Mršević, I. L. Reilly and M. K. Vamanamurthy, 'Convergence, precompactness and symmetry in quasiuniform spaces', Math. Japon. 38 (1993), 239-253.

[14] H. P. A. Künzi and S. Romaguera, 'Spaces of continuous functions and quasiuniform convergence', Acta. Math. Hungar, 75 (1997), 287-298.

[15] _ 'Completeness of the quasiuniformity of quasiuniform convergence', Ann. New York Acad. Sci. 806 (1996), 231-237.

[16] H. P. A. Künzi and C. Ryser, 'The Bourbaki quasiuniformity', Topology Proc. 20 (1995), 161-183.

[17] E. A. Michael, 'Topologies on spaces of subsets', Trans. Amer. Math. Soc. 71 (1951), 152-182.

[18] S. A. Naimpally, 'Function spaces of quasiuniform spaces', Indag. Math. 27 (1966), 768-771.

[19] B. K. Papadopoulos, 'Quasiuniform convergence on function spaces', Questions Answers Gen. Topology 12 (1994), 121-131.

[20] I. L. Reilly, P. V. Subrahmanyam and M. K. Vamanamurthy, 'Cauchy sequences in quasi-pseudometric spaces', Monatsh. Math. 93 (1982), 127-140.

[21] S. Romaguera, 'On hereditary precompactness and completeness in quasiuniform spaces', Acta. Math. Hungar. 73 (1996), 159-178.

[22] M. Seyedin, 'On quasiuniform convergence', in: General topology and its relations to modern analysis and algebra, IV (1976), pp. 425-429.

[23] R. E. Smithson, 'Topologies on sets of relations', J. Natur. Sci. Math. 11 (1971), 43-50.

[24] _ _ ' 'Uniform convergence for multifunctions', Pacific J. Math. 39 (1971), 253-259.

Department of Mathematics

The University of Auckland

Private Bag 92019

Auckland 1

New Zealand

Escuela de Caminos

Departamento de Matemática Aplicada

Universidad Politécnica de Valencia

46071 Valencia

Spain
Department of Mathematics

The University of Auckland

Private Bag 92019

Auckland 1

New Zealand 